\title{
A Novel Deep Learning Approach for Detection of Pneumonia from Chest X-rays
}

Andrew Yuan from Lynbrook High School

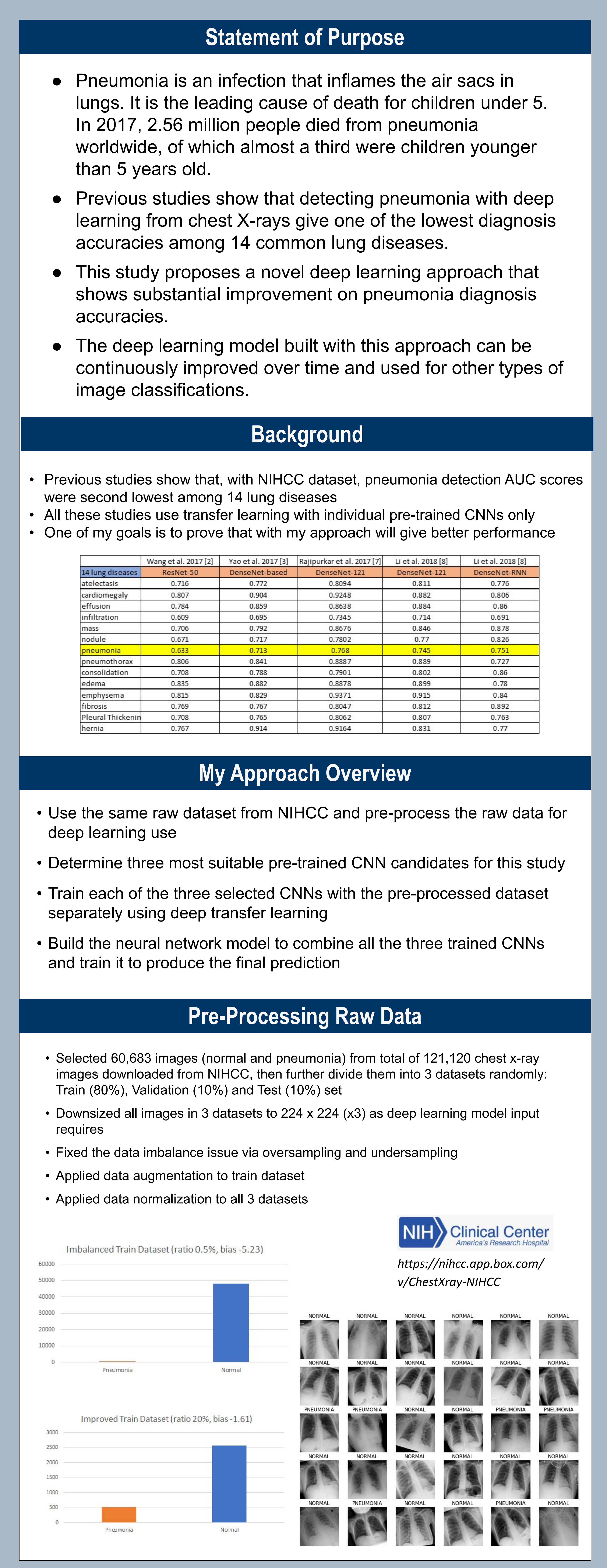

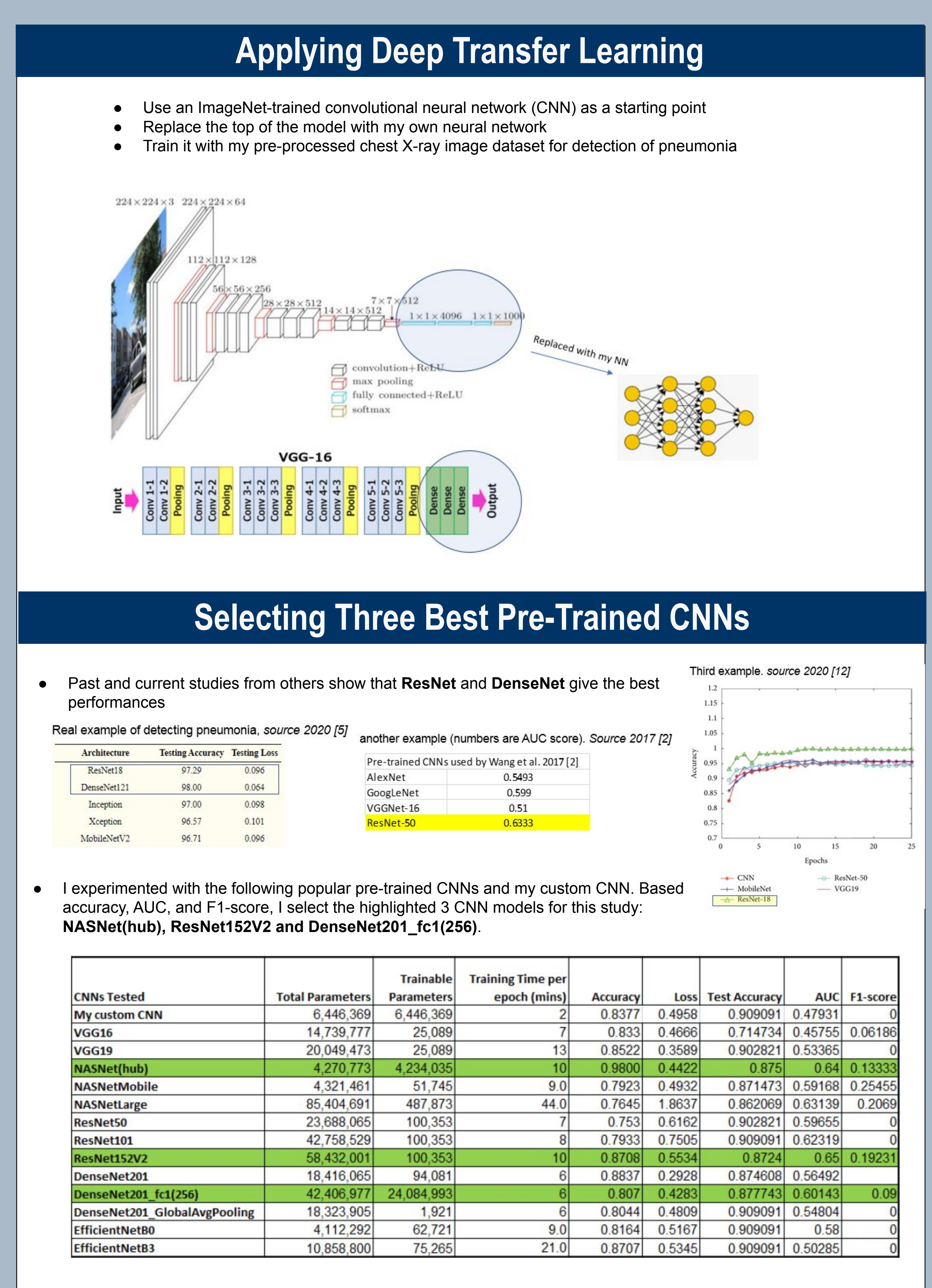

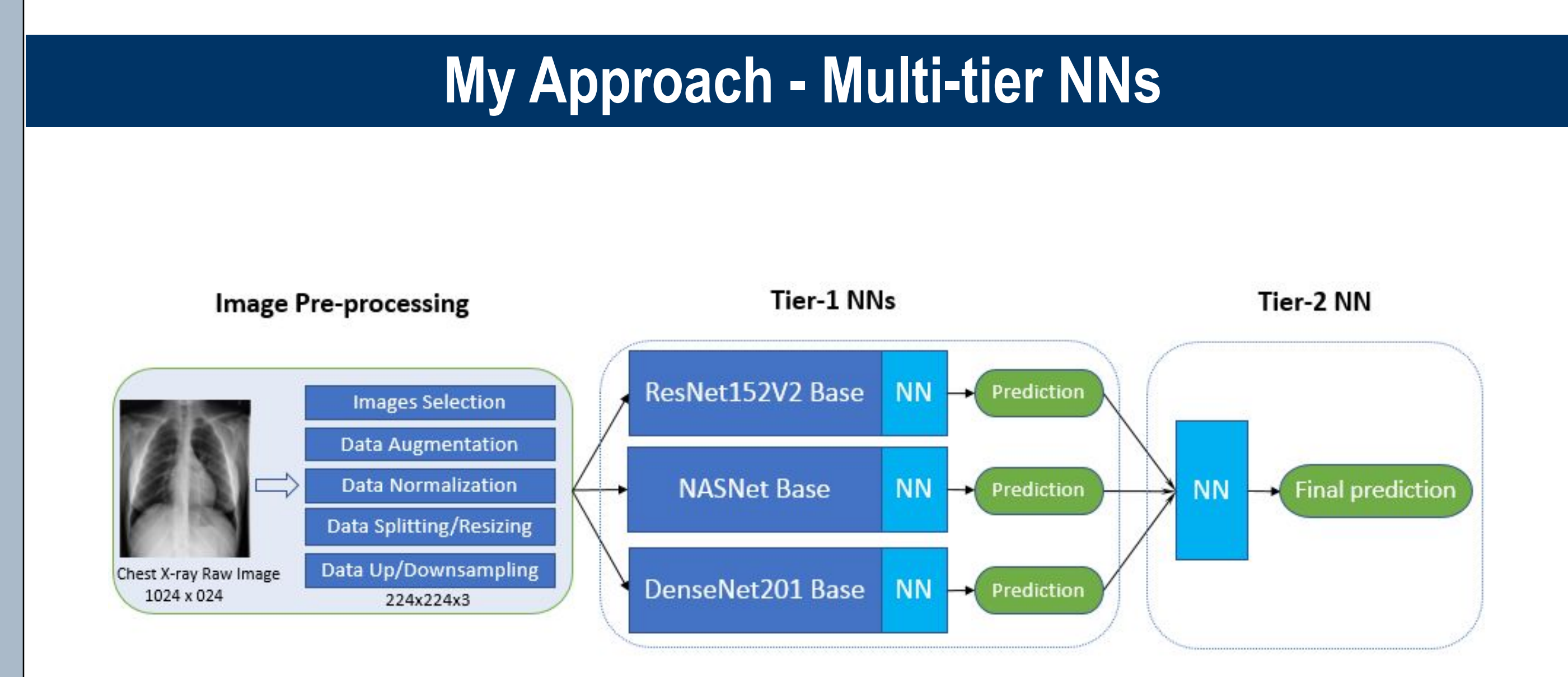

Training Tier-1 Individual NN Models Separately

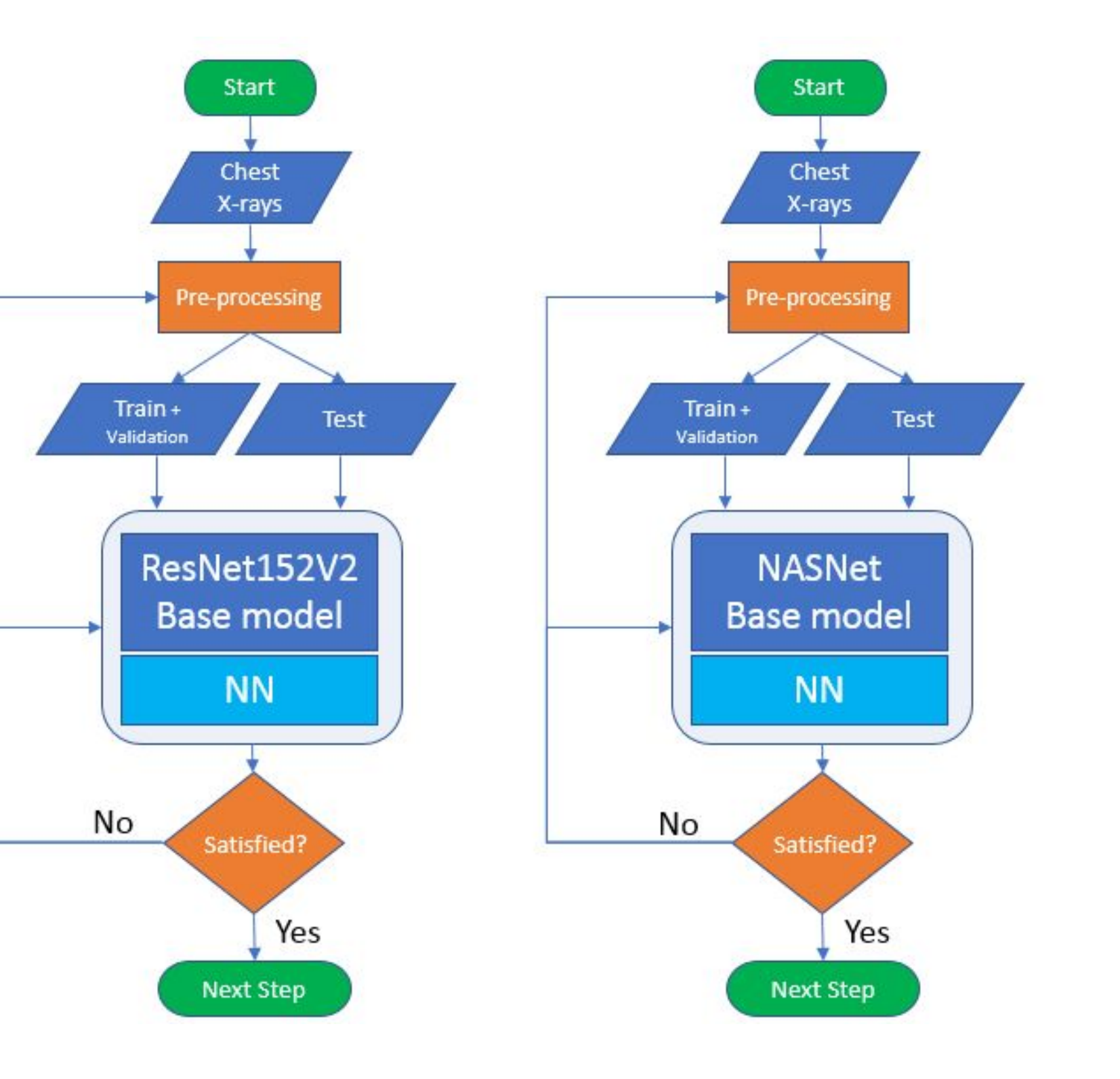

Training Tier-2 NN with Well-trained Tier-1 NNs

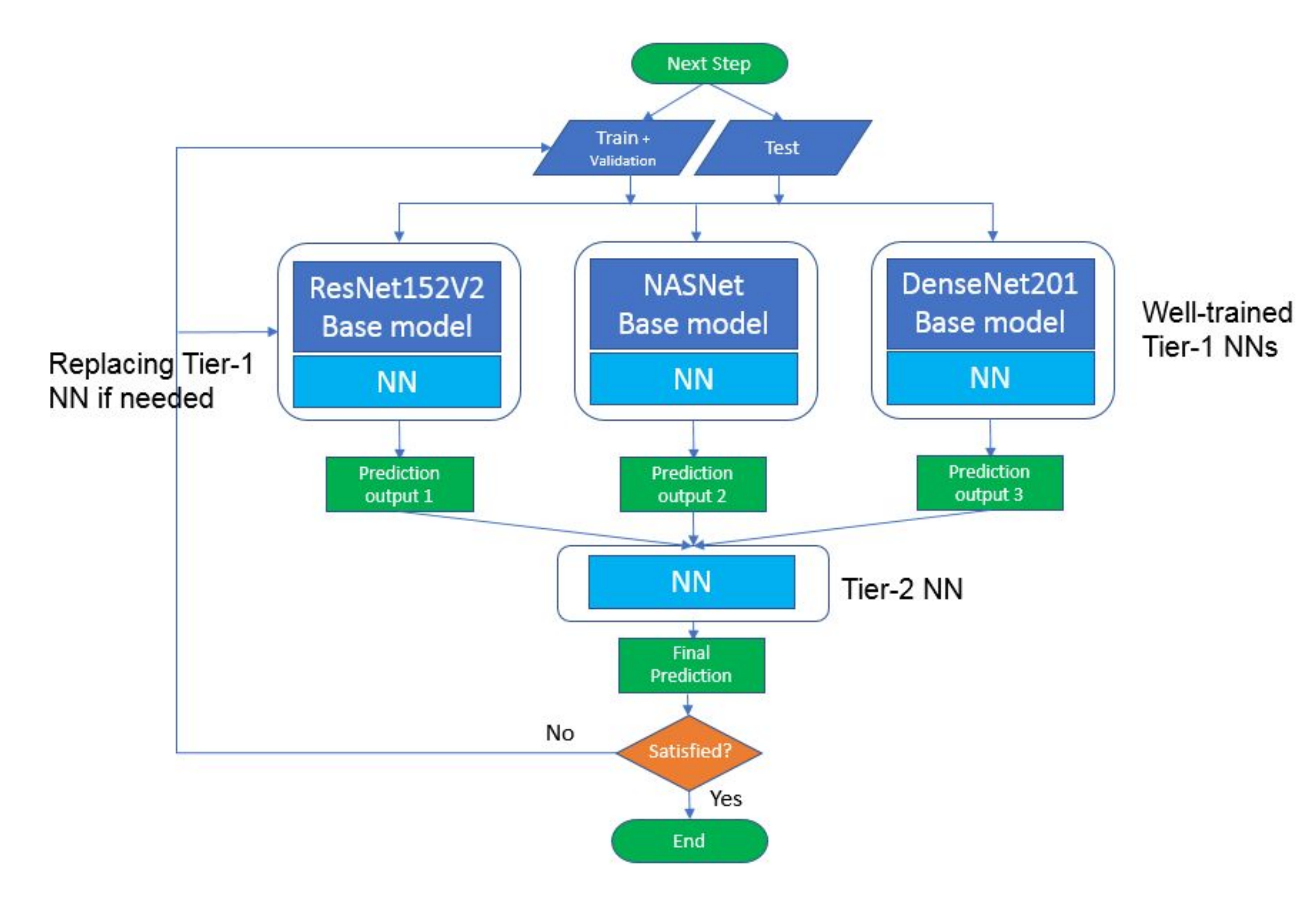

Prediction Results of Tier-1 NNs

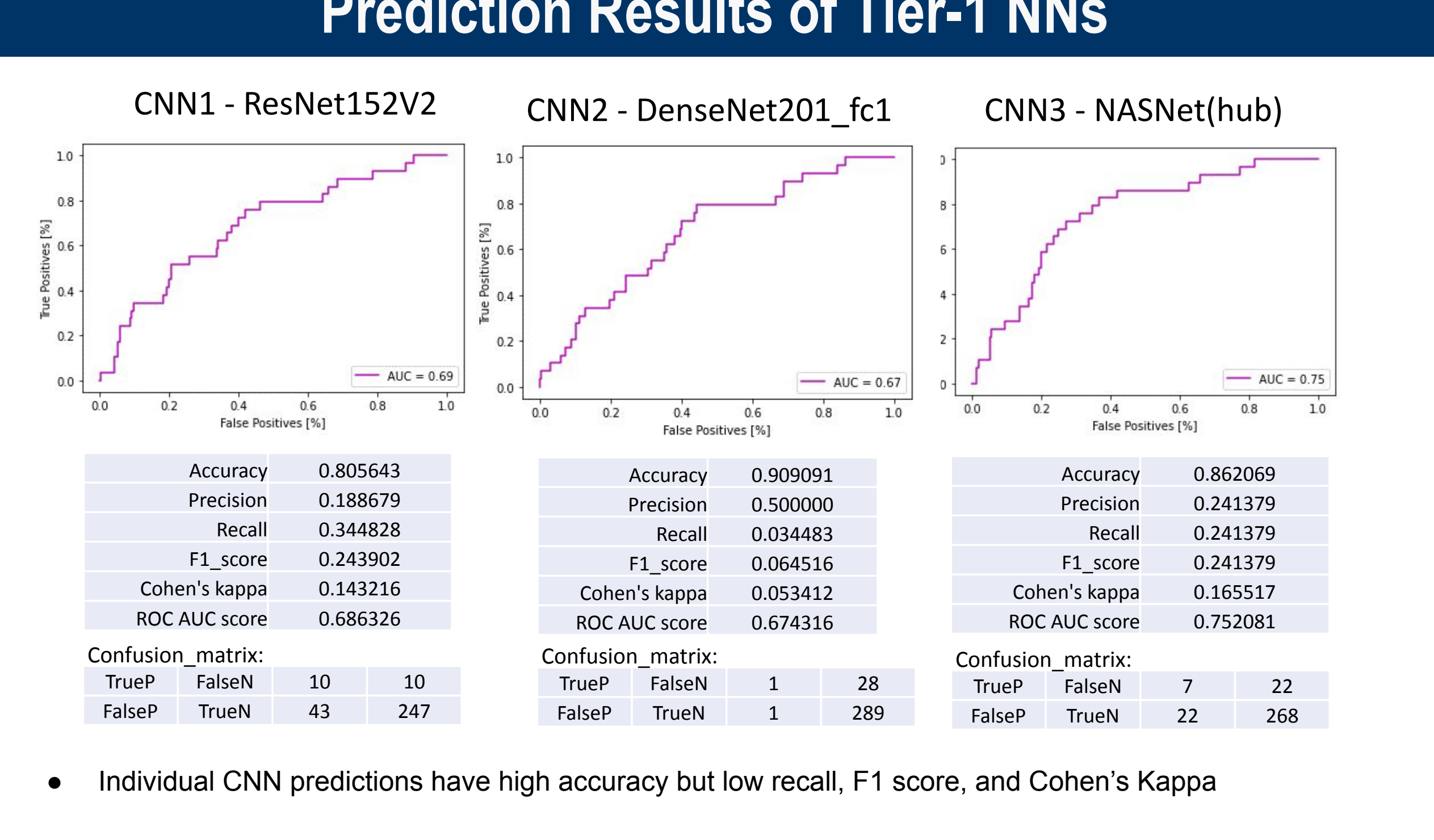

inal Prediction Results from Tier-2 NN

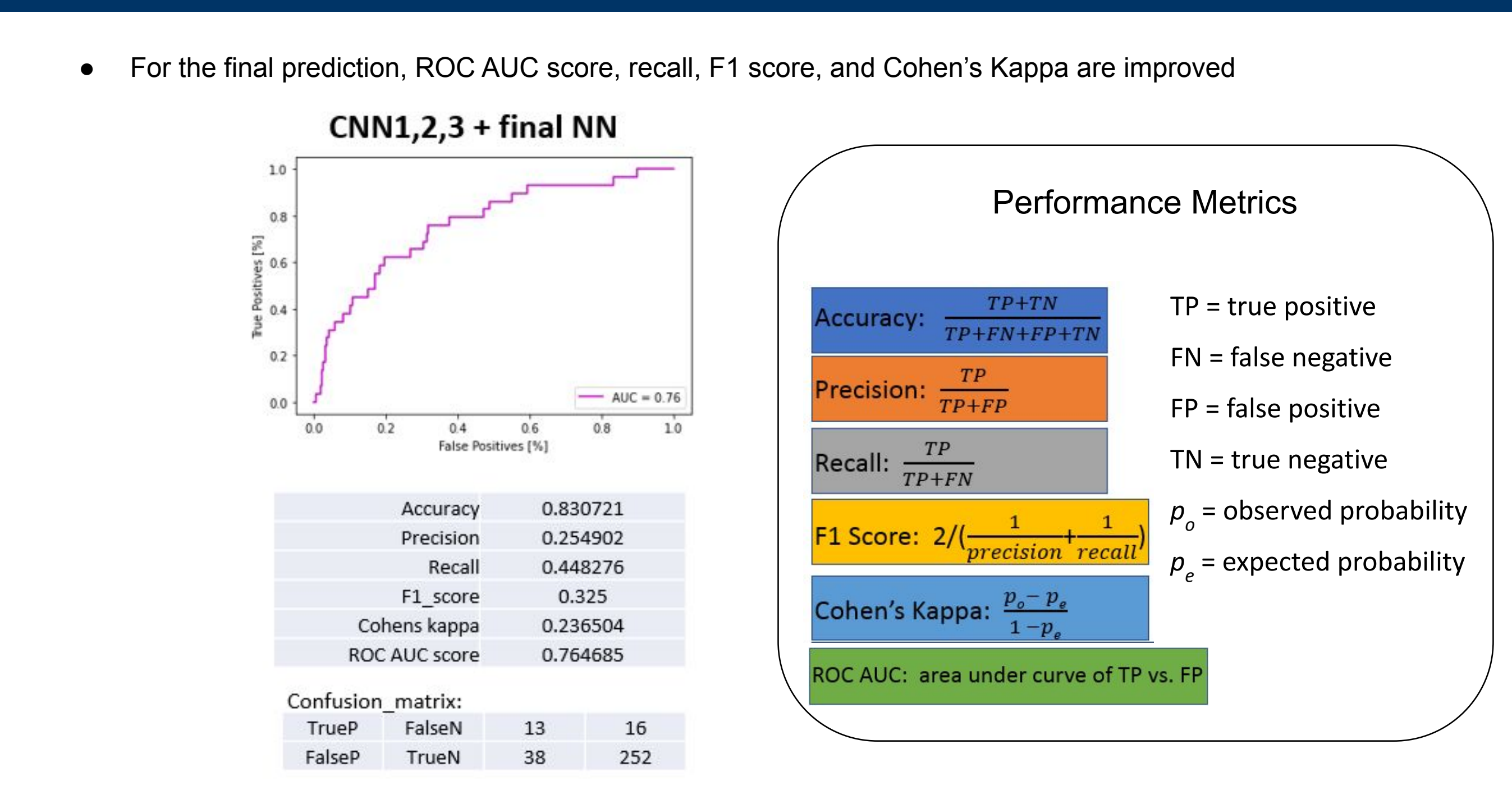

Final Prediction Results vs. Individual Tier-1 NN Results

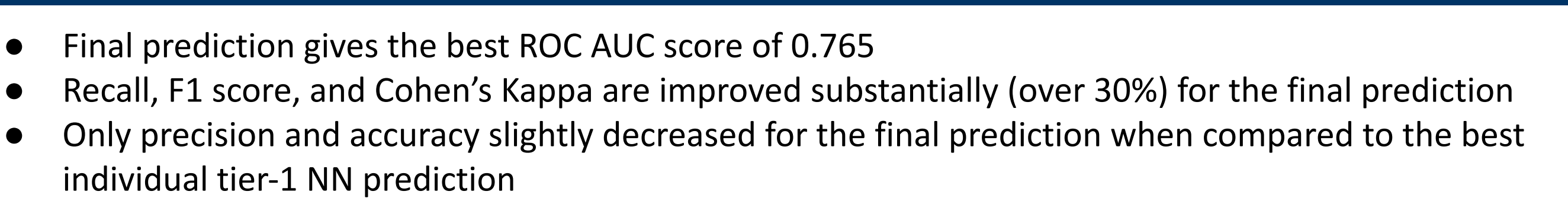
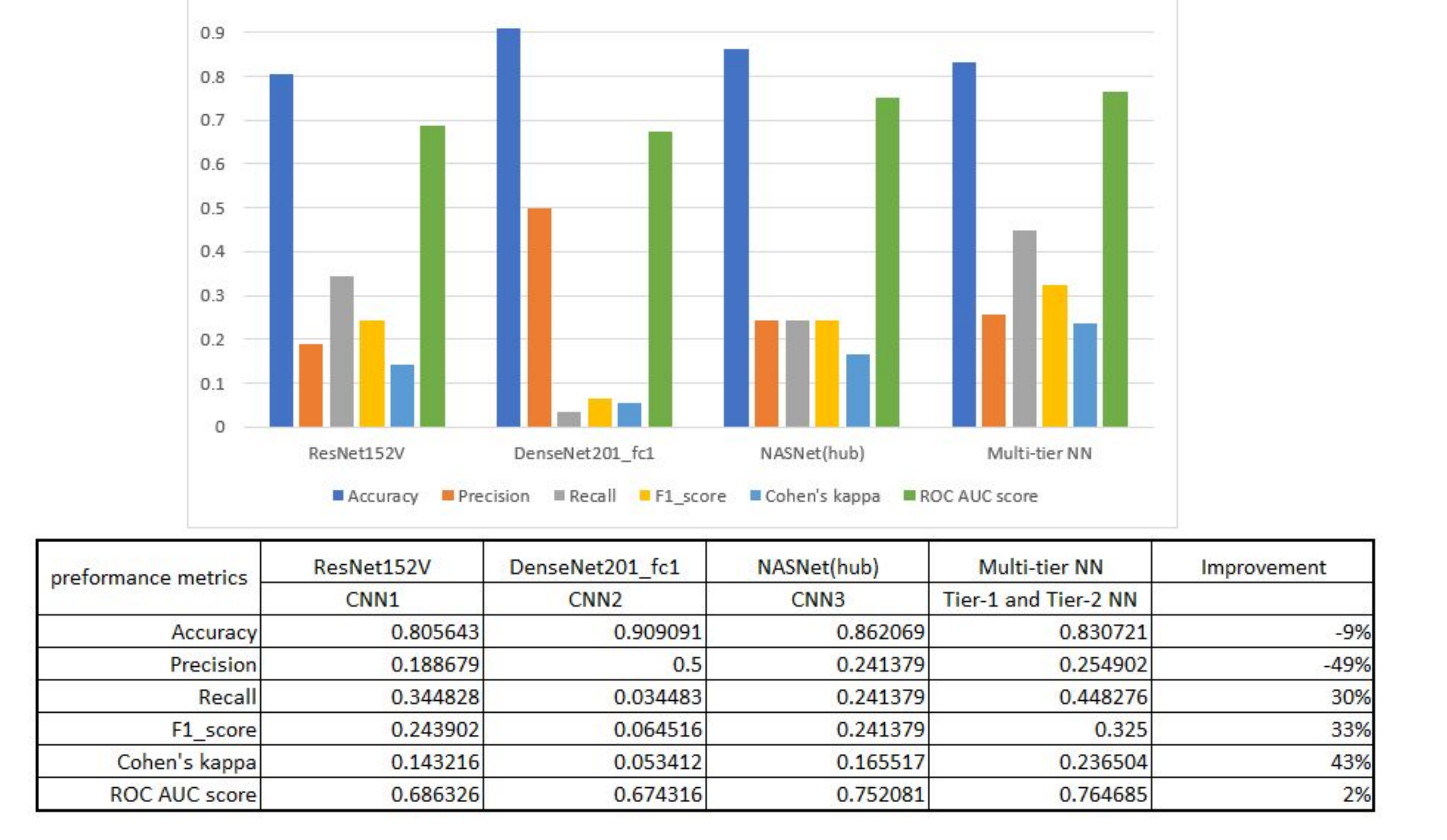

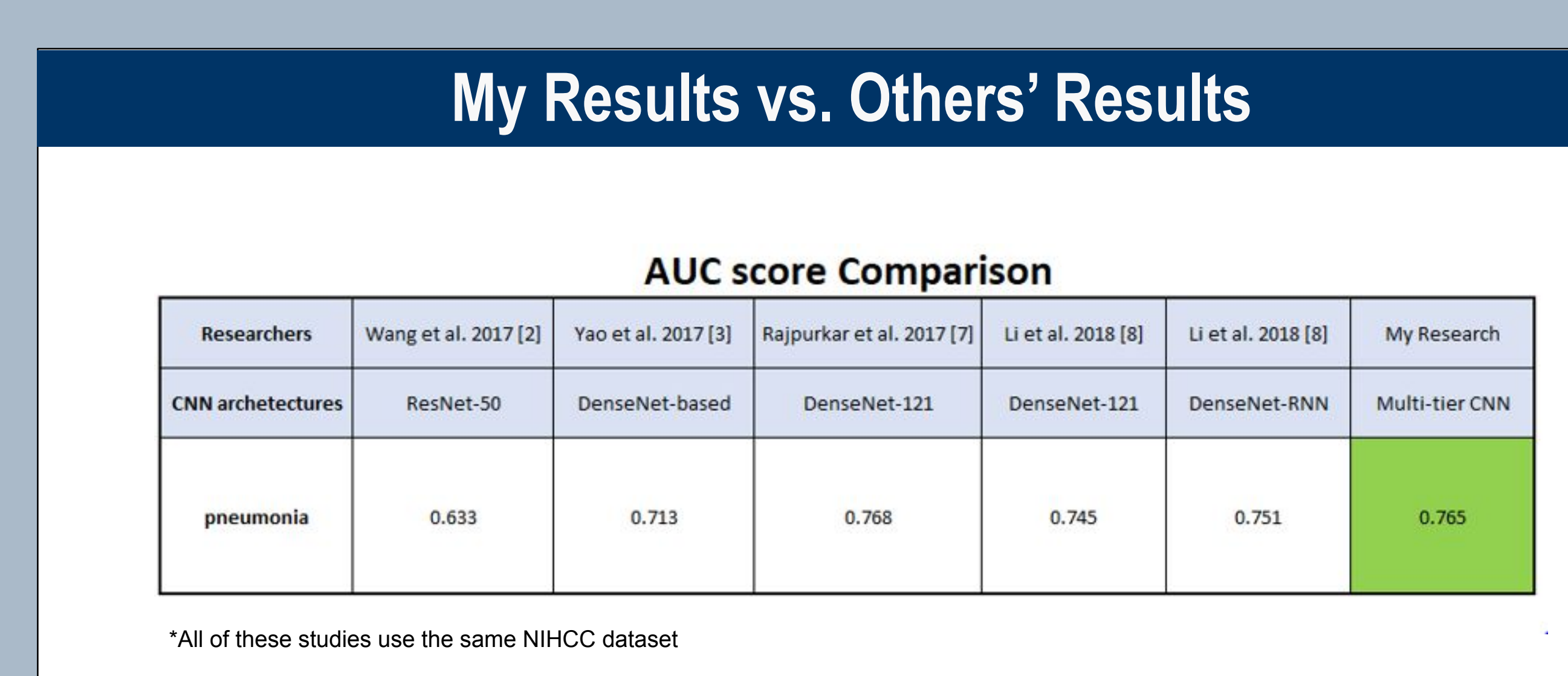

- My AUc score of 0.765 is on par with Rajpurkar et al 201770
than all other four which are $0.633,0.713,0.745,0.751$.

My Model Supports Incremental Learning

- My multi-tier NN model employs incremental learning - It can be continuously improved over time by adding more accurate tier-1
NNs or replacing the existing tier-1 NNs with better performed CNNs and re-training the Tier-2 NN after replacement

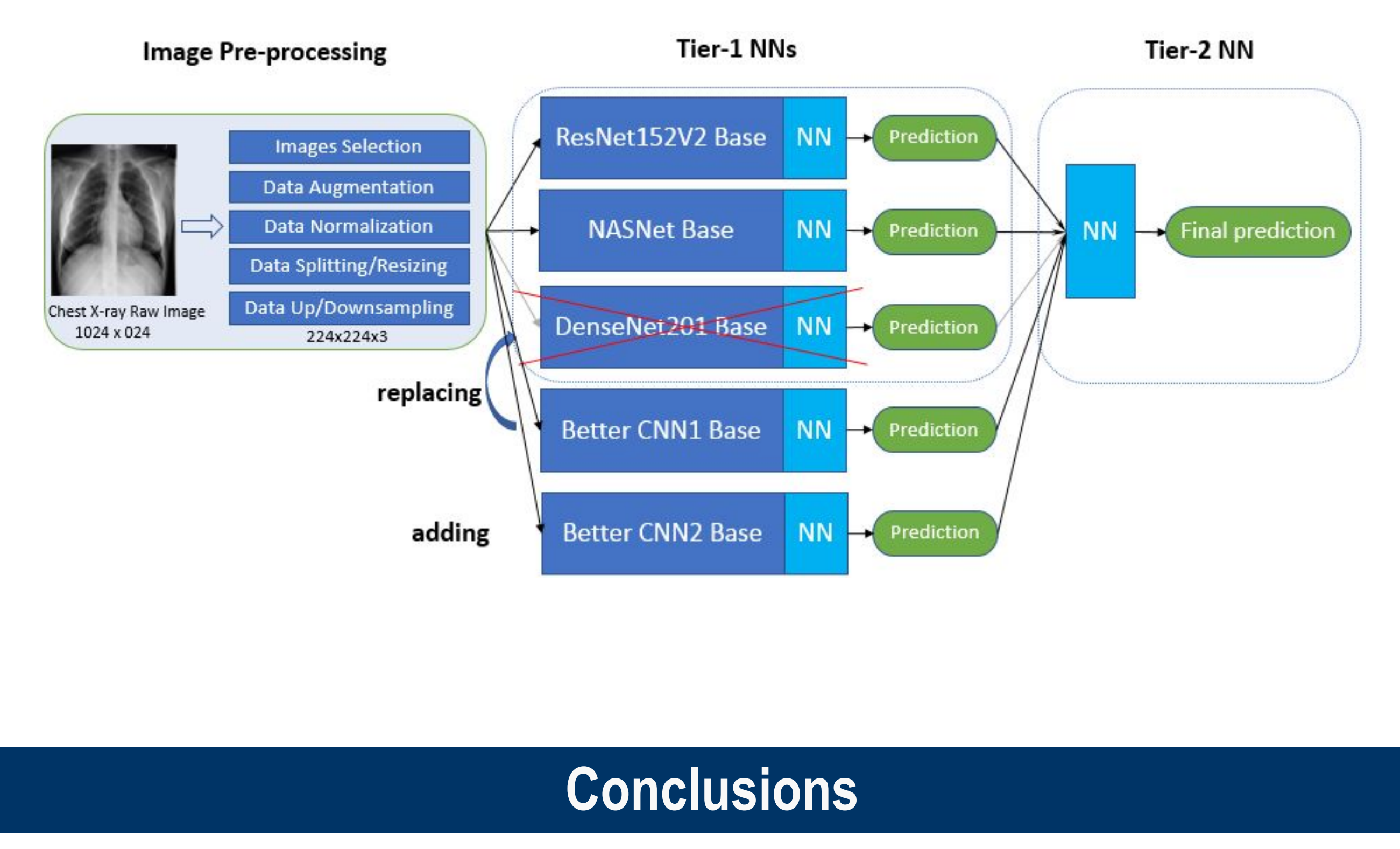

- With my multi-tier deep learning approach, I achieved an AUC of $76.5 \%$, on par or better than other past and current research results

- Compared to my individual tier-1 NNs' results, my final prediction gives an overall better performance - Myplementation. It can be continuously improved over time.

\section{Further Research}

- Extend my model from binary classification to multiclass classificatio

- Apply the new model for detection of lung cancer and other lung diseases

\section{Acknowledgement}

- Mr. Lester Leung as the mentor from Lynbrook High School who helped me with logistics and mentored me for this project - $\mathrm{NIH}$ Clinical Center for providing the raw chest $\mathrm{X}$-ray images* 